http://dx.doi.org/10.35381/r.k.v6i4.1502

\title{
Storytelling como estrategia de enseñanza en valores
}

Storytelling as a teaching strategy in values

\author{
Christian Paul Chávez-Carrera \\ christian.chavez.51@est.ucacue.edu.ec \\ Universidad Católica de Cuenca, Azogues \\ Ecuador \\ https://orcid.org/0000-0001-5971-7784 \\ Juan Carlos Erazo-Álvarez \\ jcerazo@ucacue.edu.ec \\ Universidad Católica de Cuenca, Cuenca \\ Ecuador \\ https://orcid.org/0000-0001-6480-2270 \\ Mariano Ignacio Herrera-Pérez \\ mariano.herrera@ucacue.edu.ec \\ Universidad Católica de Cuenca, Cuenca \\ Ecuador \\ https://orcid.org/0000-0002-7937-507X \\ José Antonio Pijal-Rojas \\ jose.pijal@ucacue.edu.ec \\ Universidad Católica de Cuenca, Cuenca \\ Ecuador \\ https://orcid.org/0000-0001-5348-2427
}

Recepción: 30 de agosto 2021

Revisado: 20 de septiembre 2021

Aprobación: 15 de noviembre 2021

Publicación: 01 de diciembre 2021 
Revista Arbitrada Interdisciplinaria KOINONIA

Año VI. Vol VI. N4. Edición Especial: Educación III. 2021

Hecho el depósito de Ley: FA2016000010

ISSN: 2542-3088

FUNDACIÓN KOINONIA (F.K). Santa Ana de Coro. Venezuela.

Christian Paul Chávez-Carrera; Juan Carlos Erazo-Álvarez; Mariano Ignacio Herrera-Pérez; José Antonio Pijal-Rojas

\title{
RESUMEN
}

Este analiza el uso de estrategias innovadora como lo es la narrativa digital y por medio del Storytelling la enseñanza en valores para afianzar y desde una propuesta más interactiva, dinámica e innovadora. Este trabajo ha sido fundamentado en base a investigación bibliográfica detallada en el marco teórico acerca de las narrativas digitales y uso en una nueva era digital para que pueda ser aprovechada al máximo y por qué no así en el ámbito educativo como se pretende a través de una investigación no experimental, con un enfoque cuantitativo de cohorte transversal. Para la investigación se consideró un muestreo aleatorio simple en el cual, se determinó un tamaño de muestra de 31 estudiantes. Este tipo de estrategias educativas inspiran a la generación de conocimiento en los procesos educativos, lo cual motiva tanto a docentes como a estudiantes, sin importar el nivel de educación sea: inicial, media y superior.

Descriptores: Literatura de ficción; escritura creativa; tecnología educacional. (Palabras tomadas del Tesauro UNESCO).

\begin{abstract}
This analyzes the use of innovative strategies such as digital narrative and through Storytelling the teaching of values to strengthen and from a more interactive, dynamic and innovative proposal. This work has been based on detailed bibliographic research in the theoretical framework about digital narratives and use in a new digital era so that it can be used to the maximum and why not in the educational field as intended through a non-experimental research, with a quantitative cross-sectional cohort approach. For the research, a simple random sampling was considered in which a sample size of 31 students was determined. This type of educational strategies inspire the generation of knowledge in educational processes, which motivates both teachers and students, regardless of the level of education: initial, intermediate and higher.
\end{abstract}

Descriptors: Fiction; creative writing; educational technology. (Words taken from the UNESCO Thesaurus). 


\section{INTRODUCCIÓN}

La globalización y la evolución de la comunicación y las tecnologías de la de información a nivel mundial, el uso de la tecnología no ha tenido una trasformación significativa en el ámbito educativo. Debido a varios factores entre ellos las brechas tecnológicas entre los docentes y lo estudiantes, por otra parte según un estudio realizado por la (UNESCO, 2013), el acelerado desarrollo de las TIC en la actualidad obliga a los sistemas educativos a realizar cambios en sus modelos de enseñanza con el fin de conseguir una educación acorde al desarrollo de la tecnología, proponiendo que la enseñanza sea efectiva, flexible y personalizada que les permita a los estudiantes el desarrollo de competencias, habilidades, crecimiento y formación académica que pueda desenvolverse en el mundo actual.

La importancia de las narrativas digitales en el aprendizaje tiene una gran connotación en la actualidad ya que el uso de las mismas genera y despierta interés en el público con el fin de aprender, optimizar sus conocimientos y dominio de sus aptitudes, se ha visto demostrado que en varios ámbitos y entre ellos el educativo el uso de la tecnología es fundamental para el acceso a la información de una manera mucho más eficaz y eficiente.

Como hemos analizado en el apartado anterior, los espacios y usos de desarrollo de las Tecnología de Información y Comunicación en los entornos educativos, ha adquirido un carácter trascendental dentro del ecosistema de la educación tanto local como global. A continuación, referiremos algunos aportes teóricos de acuerdo a dos ejes: en primer lugar, según las herramientas de aprendizaje y después, de acuerdo a los diversos instrumentos organizacionales dirigidos a la rama docente y cuerpo estudiantil.

Debemos empezar señalando la importancia del uso de estrategias innovadoras adecuadas al tiempo y al contexto actual, enfocando la educación en un cambio de paradigma por el apogeo de los dispositivos tecnológicos y la imposición de una enseñanza virtual a raíz de la crisis sanitaria global. Estos aspectos han fertilizado un terreno apto para la implementación de procesos, metodologías y estrategias capaces 
de generar una potenciación de los valores educacionales, así como de sus procesos pedagógicos.

Como señala (Galvis, 2008), el uso de las TIC coadyuva significativamente en la generación de procesos pedagógicos, así como en las mejoras de la educación de manera directa y simplificada. Estos beneficios, conllevan un aumento de productividad, mejora de relaciones interpersonales, fortalecimiento comunicacional y cooperativismo. Por tanto, queda demostrado que las TIC se han vuelto herramientas esenciales en los procesos educativos como modos y medios de potenciación de la retención cognitiva y la subsecuente calidad de educación.

En cuanto al storytelling, de acuerdo a (Echeverry-Ortiz, 2011), es preciso recalcar la importancia de la narración en los procesos educativos a lo largo de la historia, ya sea como un método para transferir conocimiento en edades remotas (para transmitir conocimiento acerca de periodos de cosecha o estrategias de caza, por ejemplo) o como un medio de generar las propias herramientas dinámicas de comunicación y educación a través de plataformas didácticas que hacen uso de todo el espectro de recursos audiovisuales.

Así, el storytelling queda definido como el arte de narrar de acuerdo a un uso práctico del lenguaje, sus diversos matices expresivos y emotivos, así como la construcción de imágenes y dinámicas narrativas, siendo posible ser utilizado no solo en el área educativa sino en la industria, la publicidad, el cine y todas aquellas ramas de la ciencia y el conocimiento donde interviene la comunicación a través del lenguaje visual, verbal u oral. Como menciona (Zambrano-Verdesoto et al. 2017), el storytelling facilita la construcción de identidad, sentido y periodos de vida; es decir, permite la transmisión de saberes de manera óptima y eficaz.

En el Ecuador, no existen muchas instituciones educativas que implementen estas estrategias dentro de su actividad académica, ya sea por desconocimiento o escasez de recursos, en el caso de centros educativos rurales. Sin embargo, es posible abarcar estas narrativas de acuerdo a los recursos disponibles conservando los mismos 
resultados de aprendizaje en cuanto a colaboración y aumento de comprensión de los procesos. Desde esta perspectiva, (Hermann, 2018), menciona que estas narrativas permiten producir originalidad en el proceso educacional, así como una dinámica capaz de aportar mayor interactividad en la transferencia de saberes.

La llegada de estas metodologías supuso un quiebre en el modelo tradicional, transformándolo en otro más inmersivo y versátil para ser aplicado en modelos pedagógicos y educativos. De tal manera, el nuevo paradigma contribuyó a un importante desarrollo de los procesos tecnológicos y educacionales de instituciones educativas tanto a nivel nacional e internacional. Por último, hay que manifestar que las instituciones educativas deben basar sus estructuras educativas en adecuados y pertinaces procesos sistemáticos.

Por otra parte, es importante resaltar que el proceso educativo no solo comprende la transmisión y abstracción de conocimientos sino además resaltar la implicación intrínseca del aspecto humano y sus componentes, es por ello que nos vemos avocados al abordaje una problemática actual considerando que a nivel mundial hay un declive general de la moral en escuelas e instituciones de educación. Esto es más evidente en la educación día a día a través de las aulas y para hacer una mayor demarcación, está siendo apoyado y practicado por algunos líderes religiosos.

Por lo tanto, (Harrel, 2010), comenta que el 74\% de los estadounidenses creía que la nación estaba en un estado de declive moral, una amplia mayoría (64\%) creía que las industrias del entretenimiento y los medios de comunicación tenían un papel importante que desempeñar en el resultado de estas estadísticas, tales cambios morales pueden reflejarse en la libertad de lenguaje y en varios aspectos de la vida en general.

En este sentido, los estudiantes, tienden a imitar todo lo que ven en los medios tanto de comunicación como de entretenimiento. De acuerdo a un informe en Uganda, África Oriental, muchas escuelas secundarias están plagadas de problemas como son vestimenta no apta, uso de lenguaje abusivo, embarazo adolescente y uso de sustancias psicotrópica como lo es la carencia de valores humanos y si bien por una parte es la 
institución familiar quien es la encargada directa en la formación y trasmisión de los mismos, la consolidación de estos deben ser parte del proceso educativo.

De tal manera, la pertinencia del presente estudio recalca la importancia de una adecuada implementación del storytelling como estrategia cuyo objetivo pretende persuadir y movilizar los aprendizajes en valores con el uso de herramientas audio visuales además de la expresión corporal en donde se concientice y se involucre directamente a todos los actores educativos utilizando este nexo tecnológico en donde se permita a los estudiantes una sana y armónica convivencia con la sociedad (Samson \& Allida, 2018). Tomando en cuenta el estado de la problemática actual, se puede establecer el foco de la investigación considerando la aplicación de la herramienta storytelling como una estrategia en la enseñanza de valores, esta es aplicada en la Unidad Educativa American School, ubicada en la ciudad de Cuenca, provincia Azuay, sector Capulispamba. En donde se desarrolla la estrategia la misma que se enfoca a una educación integral y con valores.

\section{Referencial teórico}

Existen algunos estudios asociados al desarrollo del uso de la metodología storytelling en la enseñanza de valores sociales asociados a la moral, tal es el caso de la investigación cuasi experimental realizada por (Madhawa-Nair et al. 2013), en donde se utilizaron dos métodos comparativos storytelling y un método convencional, la motivación y los logros fueron los valores asociados al caso positivo, este estudio fue desarrollado en los estudiantes de quinto año de una escuela primaria en Malasia, China. Considerando la muestra de 110 estudiantes se pudo determinar que, analizando dos escuelas, y analizando a dos grupos desde el enfoque experimental y controlado, al primer grupo se le enseño el método narrativo y así el segundo aprendería el método convencional. Se realizó por dos meses el estudio y al final para el análisis de datos se utilizó un modelo ANCOVA, los hallazgos detectaron una elevación significativa de 
interés y motivación en el grupo de aprendizaje de valores a considerar el grupo de aprendizaje convencional (Madhawa-Nair et al. 2013).

Un resultado similar se obtuvo, en la revisión de (Birhan et al. 2021), en donde se utilizó una pedagogía considerando como herramienta fundamental el storytelling, esta metodología se utilizó en un grupo de 18 escuelas en tres zonas East Gojjam, West Gojjam y Awi, ubicadas en Etiopía, aquí se analizaron muestras de 272 personas, tanto maestros como uno de los padres de los niños participaron en el estudio, se empleó un diseño mixto y se cuantificó por medio de cuestionarios, así como el uso de estadística para el análisis de datos.

Se encontró a la metodología como efectiva para la enseñanza de moral y valores, teniendo en consideración como valores fundamentales a equidad, respeto, honestidad y responsabilidad como el eje de enseñanza primordial, se puede indicar también que es primordial la enseñanza de valores a temprana edad, así por la retención y el método de aprendizaje al ser más gráfico deja mejores resultados que los métodos actuales de enseñanza.

Analizando un contexto Latinoamericano como eje principal la enseñanza por medio de cuentos multimedia a estudiantes se realizó en Perú, en un estudio realizado por León, en donde se enfocó al respeto como valor primordial debido a la temática de la violencia contra la mujer, que es un problema social fuerte que atraviesa la sociedad en América Latina, ya que los jóvenes al evidenciar día a día la violencia la normalizan convirtiéndolos en victimarios o víctimas, el objeto de estudio fue desarrollar mediante storytelling el mecanismo de enseñanza de valores.

Dentro de la sección metodológica se evaluó el diseño experimental como eje, teniendo una muestra de estudio 16 estudiantes de primaria de la Unidad Educativa Señor de los Milagros en Ayacucho, Perú, estos fueron asociados por temáticas como un cuento por sexo, por cada valor a aprender, para al final mediante un cuestionario se evaluó a respuesta de los estudiantes sobre la violencia, los valores más importantes en este eje 
fueron dignidad, honor, justicia y respeto. Se obtuvieron resultados positivos en el uso de esta metodología de enseñanza (León Ticlla, 2020).

Otro estudio, realizado por (Candia-Santos y Ochochoque-Carcausto, 2017), se realizó en Punó, Perú, donde se tuvo como punto principal el análisis de la narración, para la formación de los estudiantes en valores, aquí se establecieron dos variables, la dependiente la cual sería primordial como la enseñanza de valores, y, la independiente que sería el storytelling. Se analizó con niños de segundo grado de igual forma con un enfoque a) orientado a un grupo experimental con el uso de la narración, y un grupo b) un grupo de control en el cual se utilizó la metodología tradicional. En esta práctica el $80 \%$ de los niños se detectó con resultados favorables, es decir que mantuvieron la práctica y la aplicación de valores enseñados con esta estrategia, fortaleciendo y apoyando a la herramienta de narración como un eje interesante para la enseñanza de valores morales, por otro lado, el $90 \%$ de los alumnos, tuvieron una buena práctica de convivencia, con una mejor formación de valores éticos.

Por otro lado, la moral se resume en los principios y los lineamientos, así como estándares en la sociedad para comparar lo que es bueno o malo según el entorno o las personas involucradas. Actualmente, existe una decadencia moral, existen varios sucesos realizados por adolescentes o estudiantes como el crimen, violencia, bullying, abuso de drogas entre otros. Por ejemplo, las bandas delictivas condicionan a la población a salir, o los problemas que se dan a nivel social, como asesinatos, robos, un sistema de salud deficiente y un sistema de rehabilitación social deplorable (Samson \& Allida, 2018).

Si bien es cierto, es en núcleo familiar donde se encuentran la mayor parte de los principios ya que es donde en el día a día se evidencian los comportamientos y se da el establecimiento de los valores que un ser humano aprenderá para su vida, es obvio que la aplicación de estos valores se produce en el colegio o en una institución de educación, de esa forma el individuo se desenvuelve mostrando así la parte conductual y actitudinal 
ya que en un compartir con otros pares, es importante para un desarrollo como individuo y comunidad (Moreno Jácome et al. 2020).

Un individuo se desenvuelve en función del contexto familiar, esto está ligado con su personalidad y su forma de adaptarse en un entorno global, por ello la importancia de la escuela ya que está en base a la responsabilidad y coordinación tiene un objeto de carácter académico, es decir la educación se desarrolla en función a los hábitos y la aplicación como la práctica de valores.

En este sentido, se genera la educación en valores se apoya en esfuerzos deliberado por desarrollar un buen carácter basado en virtudes fundamentales que son buenas para el individuo y para la sociedad. Así como en conjunto con los padres y los miembros de la comunidad ayudan a los niños y jóvenes con sus principios y con de la mano de responsabilidad (Sari, 2013). Como un vínculo entre los conceptos de liderazgo moral y liderazgo transformacional, conecta las implicaciones sociales ligado a ambos conceptos, ya que están promulgados por medio de las relaciones sociales y tiene como objetivo principal alcanzar un nivel superior de fundamento moral.

La Constitución del Educador contempla mediante el nexo directo con el Ministerio de Educación dentro de sus planes estratégicos, por ejemplo, los planes en contra de la violencia en el sistema educativo, en este caso, se plantea dentro del Plan del Buen Vivir una educación estructurada en valores, así como tiene propuestas para mejorar la calidad del sistema educativo, planteando en la en el plan de prevención de acoso ideas como talleres de valores o los temas referentes a enseñanza en valores tanto a los estudiantes como a sus padres.

Además, dentro de la legislación, en referencia a la (Ley Orgánica de Educación Intercultural LOEI), el Art. 27 de la Constitución de la República establece Considerando que la educación debe centrarse en el ser humano y garantizará su desarrollo integral, en el marco del respeto de los derechos humanos, un medio ambiente sostenible y la democracia; Será participativo, obligatorio, intercultural, democrático, inclusivo y diversificado, de calidad y calidez; promoverá la igualdad de género, la justicia, la 
solidaridad y la paz; estimulará el sentido crítico, el arte y la cultura física, la iniciativa individual y comunitaria, el desarrollo de habilidades y habilidades para crear y trabajar Teniendo en cuenta las herramientas para poder llevar el proceso de enseñanza aprendizaje de manera sostenible, se utilizan herramientas novedosas, para poder captar la atención de padres e hijos, ya que la educación en valores va ligado a la activa participación de la familia, así como el uso de una herramienta concreta y funcional como es el storytelling. La lengua es un recurso, que es funcional por la existencia de comunidades así cada una posee sus características propias en función del idioma. El auge del storytelling y sus aplicaciones son varias, desde el área académica como en el área profesional, nace esta práctica de la narración de acuerdo a la necesidad de transmitir un conocimiento concreto a alguien, por otra parte, el storytelling es conocido como un arte, y es el hecho de contar una historia a otra persona, que en este caso es el receptor (Matus, 2019). La herramienta de storytelling ha crecido ampliamente y ha ganado popularidad al combinar imágenes, narraciones de audio grabadas, música y videos. Aunque el esta ha sido popular con un gran repunte en estos últimos años, el término fue producido por primera vez en el año 1980 por Lambert. En un contexto de aprendizaje, storytelling ha surgido en los últimos años como una poderosa herramienta de aprendizaje que involucra tanto a profesores como a estudiantes (Cherkowski et al. 2015).

La narración digital de cuentos generalmente utilizada en la educación, los niños como aprendices tienen la oportunidad de contar una historia con un componente narrativo de una actividad de aprendizaje que tiene la función de transmitir conceptos y símbolos. Aunque quizá previamente no se conocía la definición de storytelling, esta práctica ya se realizaba cuando la gente compartía historias de manera tradicional. 


\section{METODOLOGÍA}

Se empleó una investigación con diseño no experimental, con un enfoque cuantitativo de cohorte transversal (Erazo-Álvarez, 2021). Para la investigación se consideró un muestreo aleatorio simple en el cual, se determinó un tamaño de muestra de 31 estudiantes, de Primero de Bachillerato, distribuido en dos paralelos, en la Unidad Educativa American School, localizada en la Provincia del Azuay, cantón Cuenca, Panamericana Norte kilómetro 8,3.

Los datos para este estudio se recopilaron a través de una encuesta y un cuestionario para estudiantes la cual consta 8 preguntas y relacionados sobre sus valores morales, una parte de ellos son evaluados mediante la escala de Likert, fue validada a través del coeficiente de fiabilidad alfa de Cronbach de 0.756. El tiempo estimado para su desarrollo fue de 6 minutos realizado mediante la plataforma Forms de Microsoft Office, para el proceso y análisis de datos se aplicó técnicas de análisis descriptivo mediante el software estadístico SPSS.

\section{RESULTADOS}

Con referencia al análisis de pruebas de Normalidad todas las variables de la investigación dieron como resultado que son paramétricas o normales en referencia a Shapiro-Wilk además para su interpretación se utilizó las pruebas del chi cuadrado y descriptiva 


\section{Tabla 1.}

Relación entre tolerancia y amistad entre estudiantes.

\begin{tabular}{|c|c|c|c|c|c|c|}
\hline & & \multicolumn{4}{|c|}{$\begin{array}{c}\text { Me llevo bien con los estudiantes que son } \\
\text { diferentes a mí }\end{array}$} & \multirow{2}{*}{ Total } \\
\hline & & $\begin{array}{c}\text { En } \\
\text { desacuerdo }\end{array}$ & Neutral & $\begin{array}{c}\text { De } \\
\text { acuerdo }\end{array}$ & $\begin{array}{l}\text { Muy de } \\
\text { Acuerdo }\end{array}$ & \\
\hline \multirow{4}{*}{ Tolerancia } & Muy conocido & 0 & 0 & 6 & 4 & 10 \\
\hline & Conocido & 1 & 4 & 5 & 5 & 15 \\
\hline & $\begin{array}{l}\text { Medianamente } \\
\text { Conocido }\end{array}$ & 0 & 0 & 2 & 2 & 4 \\
\hline & Desconoce & 0 & 0 & 2 & 0 & 2 \\
\hline & & 1 & 4 & 15 & 11 & 31 \\
\hline \multicolumn{7}{|c|}{ Pruebas de chi-cuadrado } \\
\hline & & \multicolumn{2}{|l|}{ Valor } & $\mathrm{gl}$ & \multicolumn{2}{|c|}{ Sig. asintótica (bilateral } \\
\hline Chi-cuadrado d & & \multicolumn{2}{|l|}{$8,442^{\mathrm{a}}$} & 9 & \multicolumn{2}{|c|}{0,490} \\
\hline Razón de veros & & \multirow{2}{*}{\multicolumn{2}{|c|}{10,854}} & 9 & \multicolumn{2}{|c|}{0,286} \\
\hline Asociación line & & & & 1 & \multicolumn{2}{|c|}{0,650} \\
\hline
\end{tabular}

Fuente: Encuesta.

En referencia a la tabla 1 se analiza que 5 de los estudiantes encuestados responden: que se llevan bien con los estudiantes que son diferentes a mi porque conocen, identifican y practican el valor de la Tolerancia.

Por otra parte de acuerdo al a análisis de la prueba del chi cuadrado que estas dos variable "me llevo bien con los estudiantes diferentes a mi" y el valor tolerancia no existe una relación entre los dos por ende determinaríamos la necesidad de aplicar el uso del Storytelling como estrategia de aprendizaje para reforzar los valores humanos en este caso el valor Tolerancia 
Tabla 2.

Estadísticos descriptivos conocimiento de valores.

\begin{tabular}{lrrrrrr}
\hline & N & \multicolumn{1}{c}{ Mínimo } & Máximo & Media & Desv. típ. & Varianza \\
\hline Tolerancia & 31 & 1 & 5 & 4,00 & 1,033 & 1,067 \\
Respeto & 31 & 3 & 5 & 4,55 & 0,624 & 0,389 \\
Honestidad & 31 & 2 & 5 & 4,45 & 0,723 & 0,523 \\
Empatía & 31 & 2 & 5 & 3,74 & 0,965 & 0,931 \\
Responsabilidad & 31 & 3 & 5 & 4,55 & 0,768 & 0,589 \\
\hline N válido (según lista) & 31 & & & & & \\
\hline
\end{tabular}

Fuente: Encuesta.

De la tabla 2 podemos describir que del total de encuestados la media sobre el valor Tolerancia la respuesta que más se repitió fue 4 (conocido) por todos los estudiantes, por otra parte el valor de respeto alcanzo 4,55 como conocido así mismo el valor Honestidad alcanzo el 4,45 lo que se interpreta conocido el único valor" Empatía" dijeron que es medianamente conocido con una media de 3,74 y por ultimo el valor Responsabilidad alcanzaría un 4,55 que se interpreta como conocido de, de este análisis se puede determinar que el uso de la estrategia del Storytelling puede mejorar la formación en valores de manera global y especifica alcanzando todos los valores en análisis a una media de 5 que se interpreta como muy conocido. 


\section{Tabla 3.}

Gusto y apego a herramientas digitales.

\begin{tabular}{cccccc}
\hline & Frecuencia & Porcentaje & Porcentaje válido & $\begin{array}{c}\text { Porcentaje } \\
\text { acumulado }\end{array}$ \\
\hline Válidos & Muy de Acuerdo & 13 & 41,9 & 41,9 & 41,9 \\
& De acuerdo & 8 & 25,8 & 25,8 & 67,7 \\
& Neutral & 8 & 25,8 & 25,8 & 93,5 \\
& 2 & 6,5 & 6,5 & 100,0 \\
\hline
\end{tabular}

Fuente: Encuesta.

De la tabla 3 podemos describir que del total de encuestados con referencia la frecuencia acerca de la pregunta Me gustan las herramientas digitales el $67 \%$ estan de acuerdo en lo que corresponde a la prefencia del uso de las herramientas digitales las mismas que fascilitaran el proceso de enseñanza aprendizaje de los vsalores mediante la aplicación del Storytelling como estrategia de enseñanza, ya que se ven estimulados de mejor manera destacando que su uso permitiran conocimientos mas significativos por la interaccion que estas herramientas permiten, lo cual hace que exista una mejor interaccion, asimilacion e identificacion de los valores previamente aprendidos con lo cual el docente consolidara dichos valores. Por otra parte permitira romper la brecha tecnologica entre los alumnos nativo digitales y el docente

\section{PROPUESTA}

En base a los análisis desarrollados, podemos destacar que la aplicación del storytelling como estrategia de enseñanza podrá tener un alto grado de efectividad en varios áreas de la educación en este caso nuestro campo de estudio fueron los valores humanos y su conocimiento por parte de los estudiantes de primero de bachillerato de la Unidad Educativa American School, así con el uso de la implementación de la estrategia poder 
estimular e involucrar no solo el aspecto de aprendizaje si no también involucrar la parte emocional más sin embargo cabe destacar que puede ser aplicada a la práctica docente para reforzar de una manera eficaz, dinámica e innovadora por medio del uso de herramientas como son el audio, video y la narración digital además de estimular las habilidades creativas en el diseño y elaboración de guiones. Así de esta forma se puede romper con la brecha tecnológica que se puede generar entre los docentes y estudiantes nativos digitales. La presente propuesta está diseñada para ser aplicada en diferentes etapas.

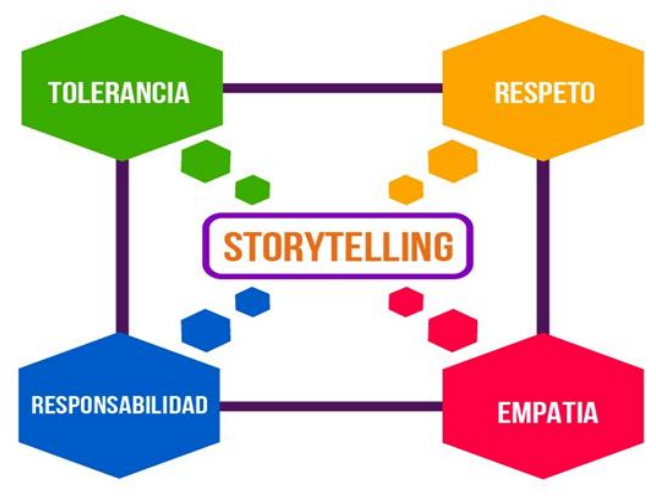

Figura 1. Storytelling y valores. Elaboración: Los autores.

Etapa1: Diseño y elaboración de un guion de tipo motivacional y que tenga a la vez una alta carga emocional y motivadora misma que llame la atención a sus pares, en donde se involucre los valores más significativos y que tenga gran relevancia para ser utilizados tanto en la vida cotidiana como en el ámbito escolar.

Etapa 2: Determinar las herramientas digitales versátiles, adecuadas e innovadoras que permitirán llegar de una manera directa y que genere la atención e involucramiento integral de los estudiantes. 
Etapa 3: Generar propuestas que permita el uso del storytelling como estrategia de enseñanza aprendizaje las cuales posean características de asequibilidad, accesibilidad, adaptabilidad y aceptabilidad, así de esta manera permitirán fomentar y afianzar cada uno de los valores empezando desde el menos conocido.

Etapa 4: Evaluar el nivel de impacto que se obtuvo por medio de generación de proyectos educativos en el cual se aplique esta estrategia del Storytelling generados por los estudiantes y orientados por los docentes de la institución American School para que a su vez puedan ser replicados a otras instituciones educativas y determinar la factibilidad de ser implementadas al interior de las aulas escolares con el fin de elevar los aprendizajes significativos con un enfoque dinámico llamativo e innovador.

\section{CONCLUSIONES}

Con base en los resultados de la investigación con referencia acerca de la gama de valores humanos en estudio si bien por una parte es la familia la principal encargada en implantar estos valores la escuela cumple el papel de dar soporte y reforzar estos valores, a través del instrumento utilizado como es la encuesta se pudo analizar y concluir que del grupo en estudio acerca de los valores mencionados la mayoría conoce el significado de los mismos y lo que se pretende es que sea muy conocidos y que puedan ser utilizados con mayor utilidad en su vida cotidiana y además se pueda comprometer de manera directa a los docentes de la Unidad Educativa ya que ellos pueden evaluar la propuesta de desarrollo del storytelling en las aulas, puesto que la considerándola como una fuente de creatividad y de llegar de una manera emocional a los estudiantes además de incorporarlo en la planificación.

Dado esto, se podra establecer un mecanismo en el cual los docentes incorporen el aprendizaje basado en valores en sus planificaciones las cuales queda instaurada la propuesta como un nuevo recurso educativo. Este tipo de estrategias educativas inspiran a la generación de conocimiento en los procesos educativos, lo cual motiva tanto a 
docentes como a estudiantes, sin importar el nivel de educación sea: inicial, media y superior.

En adición, se podrá vincular a los docentes al estudio y así puedan adquirir conocimientos para un nuevo tipo de enseñanza, en la cual destacan los valores de la creatividad. Las técnicas adquiridas permitieran la complementariedad de las actividades comunes de aprendizaje como la lectura y la escritura con actividades lúdicas en su estudio, lo cual estimula la capacidad imaginativa de los niños para poder adquirir reflexión y un criterio definido sobre diversos temas gracias a esta metodología. Estas nuevas técnicas aplicadas a la educación favorecen al aprendizaje de los grupos de estudiantes donde se apliquen, esta técnica puede ser aplicada de acuerdo a las necesidades e intereses de cada grupo.

\section{FINANCIAMIENTO}

No monetario.

\section{AGRADECIMIENTO}

A la Universidad Católica de Cuenca; por todo el apoyo brindado en la motivación y desarrollo de esta investigación.

\section{REFERENCIAS CONSULTADAS}

Birhan, W., Shiferaw, G., Amsalu, A., Tamiru, M., \& Tiruye, H. (2021). Exploring the context of teaching character education to children in preprimary and primary schools. Social Sciences \& Humanities Open, 4(1), 100171. https://doi.org/10.1016/j.ssaho.2021.100171 
Candia-Santos, L., \& Ochochoque-Carcausto, C. N. (2017). Narracion de fábulas como estrategía para la formación de valores en los niños del 2 do grado de la I.E.P N ${ }^{\circ}$ 70025 Independencia Nacional de Puno - 2017 [Storytelling of fables as a strategy for the formation of values in children of the 2 nd grade of the I.E.P No 70025 National Independence of Puno - 2017]. http://repositorio.unap.edu.pe/handle/UNAP/5568

Cherkowski, S. L., Walker, K. D., \& Kutsyuruba, B. (2015). Principals' moral agency and ethical decision-making: Towards transformational ethics. International Journal of Education Policy and Leadership, 10(5). https://doi.org/10.22230/ijepl.2015v10n5a572

Echeverry-Ortiz, A. J. (2011). Narrativas digitales: El arte de la narracion en la cibercultura [Digital Narratives: The Art of Storytelling in Cyberculture]. http://hdl.handle.net/10554/6485

Erazo Álvarez, J. C. (2021). Capital intelectual y gestión de innovación: Pequeñas y medianas empresas de cuero y calzado en Tungurahua-Ecuador [Intellectual capital and innovation management: Small and medium-sized leather and footwear companies in Tungurahua - Ecuador]. Revista De Ciencias Sociales, 27, 230-245. Recuperado a partir de https://produccioncientificaluz.org/index.php/rcs/article/view/37004

Galvis, Á. (2008). La Piola y el desarrollo profesional docente con apoyo de TIC [La Piola and teacher professional development with ICT support ]. RTV Con Autorizacion de Metacursos, 46, 77. https://n9.cl/kv6g

Harrel, A. (2010). Religion, rewards, and prosocial behavior [Religión, recompensas y comportamiento prosocial]. Recuperado de https://n9.cl/8s0n9

Hermann, A. (2018). Innovación, tecnologías y educación : las narrativas digitales como estrategias didácticas [ Innovation, technologies and education: digital narratives as didactic strategies. Revista Killkana Socialeslkana Sociales, 2(2), 31-38. https://doi.org/10.26871/killkana social.v2i2.295 
León Ticlla, M. (2020). Aplicación Web de Enseñanza de Valores mediante Cuentos Multimedia para Mejorar la Conciencia sobre la Violencia contra la Mujer en Preadolescentes Ayacucho, 2019 [Web Application of Teaching Values through Multimedia Stories to Improve Awareness about Violence against Women in Preadolescents Ayacucho, 2019]. http://repositorio.udaff.edu.pe/handle/20.500.11936/168

Ley Orgánica de Educación Intercultural LOEI. Recuperado de https://n9.cl/1fo3

Madhawa-Nair, S., Mohd -Yusof, N., \& Chooi-Hong, S. (2013). Comparing the Effects of the Story Telling Method and the Conventional Method on the Interest, Motivation and Achievement of Chinese Primary School Pupils. Procedia - Social and Behavioral Sciences, 116, 3989-3995. https://doi.org/10.1016/j.sbspro.2014.01.878

Matus, P. (2019). Storytelling . Cómo crear y contar buenas historias [Storytelling. How to create and tell good stories]. Recuperado de https://n9.cl/25odu

Moreno Jácome, M. C., Ramírez Ramírez, L. N., \& Escobar Pérez, J. Z. (2020). Revisión de educación en valores para el nivel superior en Latinoamérica [Review of Values education for higher education in Latin America]. Revista Educación, 44, 32. https://doi.org/10.15517/revedu.v44i1.35636

Samson, B., \& Allida, V. (2018). Moral decline in schools: reflections on public secondary schools in iganga district. Recuperado de https://n9.cl/wu5oz

Sari, N. (2013). The Importance of Teaching Moral values to The Students. Journal of English and Education, 1(1), 154-162. https://n9.cl/wd6c9

UNESCO. (2013). Las TIC en la educación [ICT in education]. https://es.unesco.org/themes/tic-educacion

Zambrano-Verdesoto, J. G., \& Guevara-Torres, Luis Humberto Sisa-Chenche, M. J. (2017). Estrategia de enseñanza aprendizaje Storytelling (narracion) en la educacion superior [Teaching-learning strategy Storytelling (narration) in higher education]. Recuperado de https://n9.cl/6chf6 
Revista Arbitrada Interdisciplinaria KOINONIA

Año VI. Vol VI. N4. Edición Especial: Educación III. 2021

Hecho el depósito de Ley: FA2016000010 ISSN: 2542-3088

FUNDACIÓN KOINONIA (F.K). Santa Ana de Coro. Venezuela.

Christian Paul Chávez-Carrera; Juan Carlos Erazo-Álvarez; Mariano Ignacio Herrera-Pérez; José Antonio Pijal-Rojas

(C2021 por los autores. Este artículo es de acceso abierto y distribuido según los términos y condiciones de la licencia Creative Commons Atribución-NoComercial-Compartirlgual 4.0 Internacional (CC BY-NC-SA 4.0) (https://creativecommons.org/licenses/by-nc-sa/4.0/). 